\title{
Drug Use in Systemic Lupus Erythematosus (SLE) Outpatients
}

\author{
Maria Caecilia N. Setiawati ${ }^{1, *}{ }^{*}$ Ika Puspitaningrum ${ }^{2}$ Nyoman Kertia ${ }^{3}$ \\ 1,2 Stifar Yayasan Pharmasi Semarang \\ ${ }^{3}$ Faculty of Medicine, Gadjah Mada University \\ *Corresponding author.Email: caecil_nanny@yahoo.co.id
}

\begin{abstract}
The Systemic Lupus Erythematosus (SLE) is an autoimmune disease, affecting most of the patients' organ systems. The disease is considered to be a chronic disease and its effect makes unpredictable flares. According to Yayasan Lupus Indonesia (YLI), in this decade, the total amount of SLE patients is increasing. Drug use evaluation in SLE patients is needed to improve the treatment so that the patient gets the optimal outcome. The overall aim of this research is to determine the drugs given for patients with SLE and also to know the characteristics of patients with SLE. This was a descriptive study, non-experimental research, conducted from April to August 2017. The subjects had already been diagnosed with SLE for more than 3 months. Two hundred and twenty-two patients fulfilled the inclusion criteria. Most of them $(98.20 \%)$ were women, on a productive age. Only $7.66 \%$ of the patients were given no immunosuppressant. The most used was a combination of $19.82 \%$ of methylprednisolone and mycophenolate mofetil. To reduce the side effect of the prolonged use of corticosteroid drugs, $55.41 \%$ was given Calcium supplements and Vitamin D, and 30.18\% was given anti-nausea and vomiting. The average number of drugs given was six. The most drug use in SLE outpatients was corticosteroids and the physicians had already combined the therapy with Calcium, Vitamin D, antinausea vomiting to reduce its side effect. There was no correlation between age and disease duration with the total drug given to the SLE outpatients (p 0.14 and 0.60 ).
\end{abstract}

Keywords: SLE, drug use, outpatients, disease duration

\section{INTRODUCTION}

The Systemic Lupus Erythematosus (SLE) is a systemic autoimmune disease, resulting in organ damage caused by disease activity and the complication because of its treatment. SLE is characterized by periods of flares and remission [1]. SLE's prevalence is still increasing. According to Yayasan Lupus Indonesia (YLI), in this decade, the total amount of SLE patients is increasing.

Improvements have been made in pharmacotherapy nowadays, which have positively impacted the prognosis of SLE. Drug use evaluation in SLE patients is needed to improve the treatment so that the patient gets the optimal outcome as SLE is a chronic disease and their illness can damage many organs. Drug use evaluation on SLE outpatients in Indonesia has already been done [2] but this research could get more outpatients.

This research wants to evaluate the prescribing pattern in SLE outpatients. By conducting this research, it is expected that people know more about SLE, and especially the SLE outpatients can improve their knowledge about their therapy.

\section{METHOD}

\subsection{Study design}

This was a descriptive study, non-experimental research, conducted from April to August 2017. The subjects had already been diagnosed with SLE for more than 3 months. The survey was approved by the Ethical Committee of Public Health Department, Diponegoro University No $004 / \mathrm{EC} / \mathrm{FKM} / 2015$ as research in composing a Pharmacist's Counselling Module for SLE patients.

\subsection{Material and Subject}

Medical Prescriptions from SLE patients and Questioner sheets for Subjects data. SLE outpatients who were controlled in Hospitals from several cities in Indonesia and agreed to give their data and prescription were recruited in this study. The number of SLE patients in Indonesia was limited, so the authors did not use the sampling technique. Data were descriptively analyzed and processed with SPSS 19.

\section{RESULTS}

Of the SLE outpatients from many Hospitals in Indonesia, only 222 agreed to be subjects in this research. In Table 1, it is shown that $98.20 \%$ of subjects are women, on productive age because SLE is a disease that attacks 
women more than men from any ages but almost 10-50 years [3]. There is interaction about genetic factors, estrogen (especially on 'hormone replacement therapy' but not as contraceptive used) and environment such UV from sunlight that could trigger inflammation response, induce antibody formation, and SLE [4].

In Table 1, it is shown that the mean disease duration was 6.09 years. This data showed that SLE is a chronic illness, correspond to Gordon and Isenberg, 2016. According to Doria et al, 2006, the survival rates of SLE patients at 5, 10, and 15 years after the diagnosis were $96 \%$, $93 \%$, and $76 \%$. It is that long-term prognosis remains poor in SLE patients with severe manifestations, that cause its complication in another organ, such as the cardiovascular system and kidneys, if the medicines prescribed cannot suppress the increasing immune [5].

There are 5 SLE outpatients (2.25\%) who still check their health in Hospital, but have already had a remission, so the doctor does not write a prescription for all of them. Remission occurred in SLE patients [6], although prolonged complete remissions are rare in Lupus [7].

Many drugs are used by SLE outpatients (as seen in Table II) because SLE is a multisystemic autoimmune disease with many manifestations and attack the organ system of the body [8]. The SLE outpatients usually come to see more than one clinician: rheumatologist, neurologist, cardiologist, and psychiatrist because of depression. We analyze the correlation between the total amount of the drug given to the patient with their age and disease duration, but they do not have any correlation (p. 0.14 and 0.6).

\section{DISCUSSION}

Synthetic drugs are the mainstay of therapy in SLE because it is still effective and widely available. The highly unpredictable and variable course of SLE makes the doctors think, what drugs should be prescribed for the patient individually [9].

The treatment is used to suppress the overactive immune system of the patients and diminish the inflammation caused by it. It may be stronger (e.g. steroids), and milder drugs are also widely used (such as antimalarials). The intention is to reduce drugs and ultimately discontinue the drugs' use. Patients may get fluctuating lupus, but most patients can feel better for the long term and aggressive treatment may not be needed.

Many drugs were used to treat SLE, an autoimmune disease. Corticosteroids were the most, used for $86.49 \%$ of subjects (as seen on the type of drug used in Table III), so it meant that corticosteroids were still the mainstay therapy for Indonesian SLE outpatients [10].

Corticosteroids are still frequently prescribed (86.49\%) for prolonged periods in SLE patients, which leads to side effects and for a substantial part of organ damage. The assessment of a safe daily corticosteroids dose for long-term use is important and so needs further studies with a larger patient population to know [9].
Immunosuppressant drugs are used in $92.34 \%$ of SLE outpatients (as seen in Table III) because their immune system is too active, so it needs to be suppressed. The rest $(7.66 \%)$ did not use any immunosuppressant, according to the research that immunosuppressants can be stopped without Flare Trigger in Lupus Patients. Immunosuppressant shows its effectiveness and safeness for non-renal patient SLE [11].

Antimalarials could reduce the renal severeness on lupus nephritis, as an immunomodulator, antithrombotic, and antiinflammation [12]. Hydroxychloroquine (HCQ) was an important drug in SLE because of its many beneficial effects. When patients present with persistent disease activity or need higher dosages and prolonged use of glucocorticoids (GCs), additional immunosuppressants should be prescribed by the clinicians. Based on the available evidence, azathioprine and mycophenolate mofetil are the first of choice drugs that are added. The safer GC dose for chronic daily use by SLE patients is of major importance and should be the subject of further studies in large patient populations [9]. HCQ may reduce the risk of SLE patients' flares and organ damage, reduce the dosage of steroids, and prevent the thrombotic effects of antiphospholipid antibodies. The antimalarials drug is generally safe and can be prescribed for pregnant women. However, some cautions are needed to prevent retinopathy, a rare but serious complication of the prolonged use of HCQ [13].

Eighty SLE outpatients get only one immunosuppressant, 71 patients with corticosteroids, and the rest are mycophenolate mofetyl (the most) and azathioprine, chloroquine, cyclosporine, and leflunomide. As seen in Table IV, the combination of immunosuppressant and corticosteroid is the most used drug for SLE outpatients.

Calcium Supplement and Vitamin D3 are used for 121 SLE patients in $55.41 \%$. These drugs are used to reduce osteoporosis risk, the adverse effect of prolonged use of corticosteroids. American College of Rheumatology shared guidelines in 2001 recommended calcium and Vitamin D in addition to all patient who starts corticosteroid therapy [14]. Measuring the level of calcium in the blood of SLE patients is important because hypercalcemia could occur when flaring [15]. Vitamin D had an immune modulation effect, so the deficiency of Vitamin D is a risk factor not as a result of SLE. Giving Vitamin D should reduce SLE activity and also SLE morbidity and mortality [16]. Osteoporosis is the most side effect that occurs because of the long-term use of corticosteroids even in low doses [17].

Thrombosis accident happened more often in SLE patients compared to the common population, so they need the right anticoagulant agent [18] and Aspirin is the most common given to many SLE patients [19], on this research, 23 patients were given 'blood thinner', only 7 subjects use 'blood thinner' that are not aspirin, such as cilostazol, clopidogrel, and warfarin. By using another drug, this may be because of any fear about aspirin resistance, as the research from Akdogan A, 2013 who declared that many SLE patients got resistant to aspirin [20]. 
Another clinical manifestation of SLE patients is arthritis. Pain in SLE patients consists of the pain in joints (called arthritis), the pain in muscles, headaches, or abdominal pain, that is why SLE patients need a pain killer [21]. In this research, 32 SLE patients received analgesics. The most were NSAIDs which had gastrointestinal side effects, so many SLE patients got medications for a gastrointestinal disturbance. In this research, 67 patients (30.18\%) received the drug for nausea vomiting, the most are Proton pump inhibitor and $\mathrm{H} 2$ receptor blocker. These drugs are also used for corticosteroids side effects such as peptic ulcer disease.

Hematologic manifestation in SLE patients almost occurs in different ways, such as anemia, a chronic illness [22]. Inadequate Erythropoietin (EPO) production and resistance are causing anemia in SLE. Immunosuppressive agents can increase the anemia incidence in SLE because it suppresses autoantibody [23]. In this research, 53 SLE patients got medicines for increasing Haemoglobin, folic acid is the most frequently used ( 50 subjects) the other is an iron supplement and Vitamin B12. Segal et al., 2004 declared that incidences of anemia were $46 \%$ in SLE patients, but it was not caused by folic acid deficiency, but more by Vitamin B12 deficiency [24].

Cardiovascular disease can cause death in SLE patients [25]. Hypertension almost occurs in lupus patients. ACEI and ARB are the first anti hypertension's choices of SLE [26]. The patient who gets hypertension is high on cardiovascular risk, so comorbid therapy dyslipidemia should be administered and the patient receives antihyperlipidemic drugs. Hydroxychloroquine, antimalarials that are used for the treatment of SLE patients, could improve lipid profiles and should be prescribed for patients with SLE. Statins and bile acid sequestrants are typically added first for dyslipidemia to avoid atherosclerosis [27]. Its effect is on the suppression of cholesterol synthesis, but statins also have a direct effect on the endothelium, plaque formation, and thromboxane synthesis. Statins are also reported to have anti-inflammatory and antithrombotic effects in patients with antiphospholipid syndrome such as SLE patients [28]. Aspirin is used in $10.36 \%$ of patients. Aspirin is also recommended in SLE patients with cardiovascular disease, hypertension, diabetes mellitus, hyperlipidemia, and smokers [20].

Manifestation on neuropsychiatric also occurs in SLE patients, on this research, 8 patients $(3,6 \%)$ got anticonvulsant: sodium valproate and phenytoin. Younger age could be a predictor for convulsion in SLE patients [29]. An allergic reaction is common in SLE patients [30] but in this research, only 6 patients $(2.7 \%)$ receive anti-allergy drugs, such as Cetirizin and dexchlorpheniramine maleate.

In this research, only 4 SLE outpatients $(1.8 \%)$ receive oral Hypoglycemic agents and no one uses insulin. Different from Shaharir's research, 2015 in Malaysia, which states the prevalent of Steroid induce Diabetes Mellitus (SDM) is $13 \%$ and hydroxychloroquine is the potential to protect SDM [31].
Only four subjects get antibiotics, two of them are TBC patients. Using a high-dose glucocorticoid and a cytotoxic agent such as cyclophosphamide as an immunosuppressant can cause infection [32]. It is also different from Youssef et al., 2016, who declared that serious and potential infection can occur because of the long-term use of corticosteroid and dependency on the dose [33].

The prevalent of peripheral neuropathy in SLE patients is $2-18 \%$, Huynh et al., 1999, declared that $27.8 \%$ of SLE patients got peripheral neuropathy. Eight subjects get neurotropic Vitamins; B1, B6, and B12 in high doses to overcome the peripheral neuropathy disturbance [34].

Bitencourt Dias et al, 2011, declared that there were no differences between single corticosteroid and double immunosuppressants given together for inducing remission in SLE nephritis [35]. In this research, corticosteroids or immunosuppressants were used only in $37.39 \%$ SLE outpatients and in combination with other immunosuppressants as seen in Table IV Using corticosteroids together with antimalaria and another immunosuppresant, can lower corticosteroid dose so the side effect will not occur [17]. Using corticosteroid only or combined with other immunesuppressant can induce remission in podocytopathy lupus, but flare is more often in single therapy compared with combination therapy [36] $(\mathrm{Hu}$ et al., 2015).

Azathioprine and mycophenolate mofetil are the first choice of immunosuppressive and corticosteroid-sparing agents in SLE and methotrexate is an alternative, especially in patients with arthritis or arthralgia. Cyclophosphamide is traditionally used as an inducting treatment of lupus nephritis. For the maintenance therapy, azathioprine, and mycophenolate mofetil were equally effective for lupus nephritis [9].

Cyclophosphamide combined with prednisolone is widely used in acute lupus nephritis therapy. The effects of the combination of other potent immunosuppressants such as cyclosporine with prednisolone, have been evaluated and were shown a synergistically high interaction. An immunosuppressive combination has shown a successful treatment for SLE patients and led to optimal immunosuppression [37].

Azathioprine (AZA) is a valuable immunosuppressant for the treatment of multiple manifestations of systemic lupus erythematosus (SLE) [40]. In SLE, AZA is generally prescribed for active non-renal disease, using weight-based dosing with varying suggested adjustments for renal impairment. The variability is seen in both single-response and interaction data in the azathioprine studies may be explained by its variability in pharmacogenetics [41].

Drug therapy in SLE can be improved by combining therapy with antimalarials and immunosuppressive agents and reducing Corticosteroid use [9]. The combination of Corticosteroid and immunosuppressant can be seen in Table V. 


\section{CONCLUSION}

The most drug use in SLE outpatients were corticosteroids and the physicians had already combined the therapy with Calcium, Vitamin D, anti-nausea vomiting to reduce its side effect. There was no correlation of age and disease duration with the total drug given to the SLE outpatients' (p 0.14 and 0.60).

\section{LIMITATION}

This study was cross-sectional, and the pattern of drug use in SLE patients may have been affected by its indication. The other limitation is that drugs would be prescribed differently according to SLE disease activity. We don't measure the disease activity and therefore such bias may have happened. Third, we don't use the patient's medical record, only read the drug that's prescribed by the clinicians. Nevertheless, the study provides useful information in SLE's therapy, despite its limitations.

\section{AUTHORS' CONTRIBUTIONS}

MCNS carried out the studies, collecting the data, and wrote the manuscript. IP analyzed the data and helped in to draft the manuscript. NK prepared the literature review and helped conclude. All authors contributed on manuscript revision.

\section{ACKNOWLEDGMENTS}

This research received a grant from the Directorate of Research and Community Service, Directorate General of Research and Development, Ministry of Research, Technology and Higher Mycophenolic acid (MPA) is known as a potent cytotoxic immunosuppressive agent. Although MPA displays a slight antagonism in its interaction with prednisolone, the effect of this combination shows that MPA is still a very potent immunosuppressive. Mycophenolate mofetil (MMF), a mycophenolic acid (MPA) prodrug, is also efficacious in the management of lupus nephritis and useful in the treatment of autoimmune conditions because of its mechanisms of action target $\mathrm{T}$ - and B- lymphocytes [38]. Complete steroid-sparing trials are perceived where a dose of $75 \mathrm{mg}$ MPA has the same immunosuppressive effect as $100 \mathrm{mg}$ of prednisolone. Its immunosuppressive potency is remarked at therapeutic doses and may explain that this agent has been used in the treatment of lupus glomerulonephritis by suppression of IgG autoantibody formation [39]. Education of Indonesia.

The authors would like thank to the SLE outpatients who agree to become the subjects of this research.

\section{REFERENCES}

[1] Mok, C. C and Lau, C. S, Review: Pathogenesis of systemic lupus erythematosus, J. Clin Pathol; 2003, 56:481-90
[2] Setiawati MCN, Evaluasi Penggunaan Obat, Pengukuran Aktivitas Penyakit dan Pemberian Konseling Pasien Systemic Lupus Erythematosus (SLE), MFI, 2004, Vol 9 No 2, 706-789

[3] Banerjee S, Sarkar RN, Mullick OS, et al. A cross-sectional study to assess the association of systemic lupus erythematosus disease activity with levels of high sensitivity C-reactive protein. Indian J Rheumatol. 2013: 8: 61-64

[4] Dias SS and Isenberg DA, Advances in systemic lupus erythematosus, Medicine 42:3, 2014, Elsevier

[5] Doria, A., Iaccarino, L., Ghirardello, A., Zampieri, S., Arienti, S., Sarzi-Puttini, P., Atzeni, F., Piccoli, A., Todesco, S., Long-term prognosis and causes of death in systemic lupus erythematosus. Am. J. Med. 119, 700-706. DOI: 10.1016/j.amjmed.2005.11.034

[6] Drenkard, C., Villa, A., García-Padilla, C., Pérez-Vázquez, M., Alarcón-Segovia, D., Remission of Systemic Lupus Erythematosus., 1996, DOI:10.1097/00005792-19960300000005

[7] Urowitz MB, Feletar M, Bruce IN, Ibañez D, Gladman DD. Prolonged remission in systemic lupus erythematosus. J Rheumatol. 2005 Aug;32(8):1467-72. PMID: 16078321

[8] Gordon C \& Isenberg D, Systemic Lupus Erythematosus, Oxford University Press, 2016, New York, USA

[9] Tsang-A-Sjoe, M.W.P., Bultink, I.E.M., Systemic lupus erythematosus: A review of synthetic drugs. Expert Opin. Pharmacother., 2015, 16, 2793-2806. DOI: $10.1517 / 14656566.2015 .1101448$

[10] Kasturi, S., Sammaritano, L.R. Corticosteroids in Lupus. Rheum. Dis. Clin., 2016, 42, 47-62. DOI: 10.1016/j.rdc.2015.08.007

[11] Pego-Reigosa, J.M., Cobo-Ibáñez, T., Calvo-Alén, J., LozaSantamaría, E., Rahman, A., Muñoz-Fernández, S., RúaFigueroa, Í. Efficacy and safety of nonbiologic immunosuppressants in the treatment of non-renal systemic lupus erythematosus: a systematic review. Arthritis Care Res. 2013, 65, 1775-1785. DOI:10.1002/acr.22035

[12] Lee SJ, Silverman E, Bargman JM. The role of antimalarial agents in the treatment of SLE and lupus nephritis. Nat Rev Nephrol. 2011, Oct 18;7(12):718-29. DOI: 10.1038/nrneph.2011.150. PMID: 22009248.

[13] Ponticelli, C., Moroni, G., Hydroxychloroquine in systemic lupus erythematosus (SLE), Expert Opin. Drug Saf.2017, 16, 411-419.DOI: 10.1080/14740338.2017.1269168

[14] Singh, A., Kamen, D.L., Potential benefits of vitamin D for patients with systemic lupus erythematosus, Dermatoendocrinol. 2012, 4, 146-151. DOI: $10.4161 /$ derm. 20443

[15] Karageorgas, Tseronis, Mavragani, Activation of Type I Interferon Pathway in Systemic Lupus Erythematosus: Association with Distinct Clinical Phenotypes, BioMed Research International, 2011, https://doi.org/10.1155/2011/273907

[16] Pakpoor, J., Pakpoor, J., Vitamin D Deficiency and Systemic Lupus Erythematosus: Cause or Consequence? Oman MedJ.2003, 28, 295. doi:10.5001/omj.2013.83 
[17] Ruiz-Irastorza, G., Danza, A., Khamashta, M., Glucocorticoid use and abuse in SLE. Rheumatol. Oxf. Engl.,2012, 51, 1145-1153. DOI:10.1093/ rheumatology/ker410

[18] Burgos PI, Alarcón GS, McGwin G Jr, Crews KQ, Reveille JD, Vilá LM. Disease activity and damage are not associated with increased levels of fatigue in systemic lupus erythematosus patients from a multiethnic cohort: LXVII. Arthritis Rheum. 2009;61(9):1179-1186. DOI:10.1002/art.24649

[19] Wahl DG, Bounameaux H, de Moerloose P, Sarasin FP. Prophylactic Antithrombotic Therapy for Patients with Systemic Lupus Erythematosus with or without Antiphospholipid Antibodies: Do the Benefits Outweigh the Risks? A Decision Analysis. Arch Intern Med. 2000;160(13):2042-2048. DOI:10.1001/archinte. 160.13.2042

[20]Akdogan A, Kilic L, Akman U, Dogan I, Karadag O, Bilgen SA, Buyukasik Y, Kiraz S, Ertenli I. Aspirin resistance in systemic lupus erythematosus. A pilot study. Lupus. 2013 Jul;22(8):835-8. DOI: 10.1177/0961203313493487

[21] Waldheim, Eva. 2013, Self Reported Pain in SLE, Karolinka Instituted, Sweden

[22] Giannouli, S., Voulgarelis, M., Ziakas, P.D., Tzioufas, A.G., Anaemia in systemic lupus erythematosus: from pathophysiology to clinical assessment. Ann. Rheum. Dis.2006, 65, 144-148. DOI:10.1136/ard.2005.041673

[23] Ardalan, M.-R., Anemia in lupus nephritis; etiological profile. J. Ren. Inj. Prev.2013, 2, 103-104. DOI:10.12861/jrip.2013.32

[24] Segal, R., Baumoehl, Y., Elkayam, O., Levartovsky, D., Litinsky, I., Paran, D., Wigler, I., Habot, B., Leibovitz, A., Sela, B.A., Caspi, D. Anemia, serum vitamin B12, and folic acid in patients with rheumatoid arthritis, psoriatic arthritis, and systemic lupus erythematosus. Rheumatol. Int.2004, 24 , 14-19. DOI:10.1007/s00296-003-0323-2

[25] Van Leuven SI, Mendez-Fernandez YV, Wilhelm AJ, Wade NS, Gabriel CL, Kastelein JJ, Stroes ES, Tak PP, Major AS. Mycophenolate mofetil but not atorvastatin attenuates atherosclerosis in lupus-prone LDLr (-/-) mice. Ann Rheum Dis. 2012 Mar;71(3):408-14. DOI: 10.1136/annrheumdis2011-200071. Epub 2011 Sep 27. PMID: 21953346; PMCID: PMC3306213.

[26] Tselios, K., Koumaras, C., Urowitz, M.B., Gladman, D.D, Do current arterial hypertension treatment guidelines apply to systemic lupus erythematosus patients? A critical appraisal,2014 Semin. Arthritis Rheum. 43, 521-525. DOI: 10.1016/j.semarthrit.2013.07.007

[27] Ardoin, S.P., Sandborg, C., Schanberg, L.E. Management of dyslipidemia in children and adolescents with systemic lupus erythematosus. Lupus 2007, 16, 618-626. DOI:10.1177/0961203307079566

[28] Tu, H., Li, Q., Xiang, S., Jiang, H., Mao, Y., Shou, Z., Chen, J., Dual effects of statins therapy in systemic lupus erythematosus and SLE-related atherosclerosis: the potential role for regulatory $\mathrm{T}$ cells. Atherosclerosis 2012, 222, 29 33. DOI: $10.1016 /$ j.atherosclerosis.2012.02.023
[29] Andrade, R.M., Alarcón, G.S., González, L.A., Fernández, M., Apte, M., Vilá, L.M., McGwin, G., Reveille, J.D. Seizures in patients with systemic lupus erythematosus: data from LUMINA, a multiethnic cohort (LUMINA LIV). Ann. Rheum. Dis., 2008, 67, 829-834. DOI:10.1136/ard.2007.077594

[30] Wozniacka A, Sysa-Jedrzejowska A, Robak E, Samochocki Z, Zak-Prelich M. Allergic diseases, drug adverse reactions, and total immunoglobulin E levels in lupus erythematosus patients. Mediators Inflamm. $2003 \mathrm{Apr} ; 12(2): 95-9$. DOI: 10.1080/0962935031000097709. PMID: 12775359; PMCID: PMC1781602.

[31] Shaharir SS, Gafor AH, Said MS, Kong NC. Steroid-induced diabetes mellitus in systemic lupus erythematosus patients: analysis from a Malaysian multi-ethnic lupus cohort. Int J Rheum Dis. 2015 Jun;18(5):541-7. DOI: 10.1111/1756185X.12474. Epub 2014 Oct 8. PMID: 25294584.

[32] Kang, I., Park, S.H., Infectious complications in SLE after immunosuppressive therapies. Curr. Opin. Rheumatol 2003. $15,528-534$.

[33] Youssef, J., Novosad, S.A., Winthrop, K.L. Infection Risk and Safety of Corticosteroid Use. Rheum. Dis. Clin. North Am.,2016, 42, 157-176, ix-x. DOI:10.1016/j.rdc.2015.08.004

[34] Huynh, C., Ho, S.-L., Fong, K.-Y., Cheung, R.T.F., Mok, C.C., Lau, C.-S., Peripheral Neuropathy in Systemic Lupus Erythematosus. J. Clin. Neurophysiol. 1999, 16, 164-168

[35] Bitencourt Dias, C., Pinheiro, C.C., Malafronte, P., Titan, S., Alves de Brito, G., Gera Abrão, J., Dos Santos Silva, V., Toledo Barros, R., Woronik, V. Prednisone monotherapy induced remission in a group of patients with membranous lupus nephritis. Clin. Nephrol., 2011, 76, 57-63.

[36] Hu, W.X., Chen, Y.H., Bao, H., Liu, Z.Z., Wang, S.F., Zhang, H.T., Liu, Z.H. Glucocorticoid with or without additional immunosuppressant therapy for patients with lupus podocytopathy: a retrospective single-center study,2015, Lupus 24, 1067-1075. DOI:10.1177/0961203315578766

[37] Ferron GM, Jusko WJ. Species and gender-related differences in cyclosporine/ prednisolone/sirolimus interactions in whole blood lymphocyte proliferation assays. J Pharmacol Exp Ther. 1998; 286: 191-200.

[38] Sahin A. Mycophenolate mofetil in the treatment of systemic lupus erythematosus. Eurasian J Med. 2009;41(3):180-185.

[39] Chan TM, Li FK, Tang CS, Wong RW, Fang GX, Ji YL, Lau CS, Wong AK, Tong MK, Chan KW, Lai KN. Efficacy of mycophenolate mofetil in patients with diffuse proliferative lupus nephritis. Hong Kong-Guangzhou Nephrology Study Group. N Engl J Med. 2000 Oct 19;343(16):1156-62. DOI: 10.1056/NEJM200010193431604. PMID: 11036121.

[40] Okon LG, Werth VP. Cutaneous lupus erythematosus: diagnosis and treatment. Best Pract Res Clin Rheumatol 2013;27:391 404. DOI:10.1016/j.berh. 2013.07.008

[41] Siva C, Yokoyama W, McLeod H. Pharmacogenetics in rheumatology: the prospects and limitations of an emerging field. Rheumatology. 2002;41:1273- 